\title{
ON THE EXISTENCE OF POSTPROJECTIVE COMPONENTS IN THE AUSLANDER-REITEN QUIVER OF AN ALGEBRA
}

\author{
By
}

\author{
P. DräXler and J. A. de la PeÑA
}

Let $k$ be an algebraically closed field and $A$ be a basic finite-dimensional $k$ algebra of the form $A=k Q / I$, where $Q$ is a quiver (= finite oriented graph) and $I$ is an admissible ideal of the path algebra $k Q$, see [3]. In this work we assume that $Q$ has no oriented cycles.

Let $\bmod _{A}$ denote the category of finite dimensional left $A$-modules. For each indecomposable non-projective $A$-module $X$, the Auslander-Reiten translate $\tau_{A} X$ is an indecomposable non-injective module. The Auslander-Reiten quiver $\Gamma_{A}$ has as vertices representatives of the isoclasses of the finite dimensional indecomposable $A$-modules, there are as many arrows from $X$ to $Y$ as $\operatorname{dim}_{k} \operatorname{rad}_{A}(X, Y) / \operatorname{rad}_{A}^{2}(X, Y)$. In this paper we do not distinguish between a module and its corresponding isoclass. A connected component $\mathscr{P}$ of $\Gamma_{A}$ is postprojective if $\mathscr{P}$ has no oriented cycles and each module $X$ in $\mathscr{P}$ has only finitely many predecessors in the path order of $\mathscr{P}$. Several important classes of algebras have postprojective components: hereditary algebras $[3,6]$, algebras satisfying the separation condition $[1,2]$, tilted algebras [8].

The aim of this work is to find necessary and sufficient conditions for the existence of postprojective components in $\Gamma_{A}$. In section 1 we give an algorithmic procedure to decide the existence of postprojective components. In section 2 we consider a one-point extension algebra $A=B[M]$ such that all indecomposable direct summands of $M$ belong to postprojective components of $\Gamma_{B}$, then we give conditions that assure that the projective $A$-module $P$ with $\operatorname{rad} P=M$ lies in a postprojective component of $\Gamma_{A}$. In section 3 we consider some special cases. We recall that once identified a postprojective component $\mathscr{P}$ of $\Gamma_{A}$, the modules on $\mathscr{P}$ may be constructed using the knitting procedure [3]. In [5], an algorithmic procedure which makes essential use of the knitting procedure is given to construct all the postprojective components of $\Gamma_{A}$.

Received October 24, 1994.

Revised June 6, 1995. 
The research for this paper started during a stay of the first named author at UNAM, México in March 93 and it was completed during a stay of the second author at Bielefeld in May 94. Both authors thank their Institutions, DAAD (Germany) and CONACYT and DGAPA, UNAM (Mexico) for support.

\section{Existence of postprojective components.}

1.1. Let $A=k Q / I$ be a finite dimensional $k$-algebra such that the quiver $Q$ has no oriented cycles. We may consider $A$ as a $k$-category with objects the set of vertices $Q_{0}$ of $Q$ and morphisms from $x, y \in Q_{0}$ the space $A(x, y)=e_{y} A e_{x}$, where $e_{x}$ denotes the trivial path at the vertex $x$. For two vertices $x, y \in Q_{0}$ we write $y \leq x$ if there is a path from $y$ to $x$ in $Q$.

Let $x \in Q_{0}$, we denote by $A^{x}$ the full subcategory of $A$ whose vertices are those $y \in Q_{0}$ with $y \leq x$. Observe that the quiver $Q^{x}$ of $A^{x}$ is a convex (= path closed) subquiver of $Q$. The indecomposable projective $A$-module $P_{x}=A e_{x}$ has radical rad $P_{x}$ which is an $A^{x}$-module. We denote by $\operatorname{rad} P_{x}=\oplus_{i=1}^{n_{x}} R_{i}^{x}$ the indecomposable decomposition of $\operatorname{rad} P_{x}$.

1.2. A path in $\bmod _{A}$ is a sequence $\left(X_{0}, \cdots, X_{s}\right)$ of (isomorphisms classes of) indecomposable $A$-modules $X_{i}, 0 \leq i \leq s$, such that there is a map $0 \neq f_{i} \in$ $\operatorname{Hom}_{A}\left(X_{i}, X_{i+1}\right)$ which is not an isomorphism, $0 \leq i \leq s-1$. In this case we write $X_{0} \leq X_{s}$ and we say that $X_{0}$ is a predecessor of $X_{s}$. If $s=1$ and $X_{0}=X_{s}$ we say that the path $\left(X_{0}, \cdots, X_{s}\right)$ is a cycle.

Following [4] we say that a module $M$ is directing in $\bmod _{A}$ provided there do not exist indecomposable direct summands $M_{1}$ and $M_{2}$ of $M$ and an indecomposable non-projective module $X$ such that $M_{1} \leq \tau X$ and $X \leq M_{2}$. It is shown in [4] that an indecomposable module $X$ is directing if and only if there are no cycles $\left(X_{0}, \cdots, X_{s}\right)$ with $X_{0}=X=X_{s}$. The following result will be important in our work.

TheOREM $[4,7]$. Let $x \in Q_{0}$. Then $P_{x}$ is directing in $\bmod _{A}$ if and only if $\mathrm{rad}$ $P_{x}$ is directing in $\bmod _{A}$.

Moreover, if $x$ is a source, then $P_{x}$ is directing in $\bmod _{A}$ if and only if $\operatorname{rad} P_{x}$ is directing in $\bmod _{A^{x}}$.

1.3. We state our main result which provides an algorithmic criterion for the existence of postprojective components.

THEOREM. Let. $A=k Q / I$ be a finite dimensional $k$-algebra such that $Q$ has no 
oriented cycles. Then $\Gamma_{A}$ has a postprojective component if and only if for each vertex $x \in Q_{0}$ one of the following conditions is satisfied:

(1x) there is a postprojective component $\mathscr{P}$ of $\Gamma_{A^{x}}$ such that $R_{i}^{x} \notin \mathscr{P}$ for every $1 \leq i \leq n_{x}$;

(2x) for each $1 \leq i \leq n_{x}$ the set of predecessors $\left\{Y \in \Gamma_{A^{x}}: Y \leq R_{i}^{x}\right\}$ of $R_{i}^{x}$ in $\bmod _{A^{x}}$ is finite and formed by directing modules. Moreover, if $x$ is a source, then $\operatorname{rad} P_{x}$ is directing in $\bmod _{A^{x}}$.

We prove the theorem in (1.5) after some preparation. In (1.8) we give some examples.

1.4. LemmA. Assume that all $x \in Q_{0}$ the condition $(2 \mathrm{x})$ is satisfied, then $\Gamma_{A}$ has a postprojective component.

PROOF: We claim that for every $x \in Q_{0}$ the following condition is satisfied:

(3x): for each $1 \leq i \leq n_{x}$, the set of predecessors $\left\{X \in \Gamma_{A}: X \leq R_{i}^{x}\right\}$ of $R_{i}^{x}$ in $\bmod _{A}$ is finite and formed by directing modules.

Indeed, let $X$ be a predecessor of $R_{i}^{x}$ in $\Gamma_{A}$ and assume that $X$ is not an $A^{x}$ module. We may assume that $x$ is minimal with this property in the path order of $Q$. Then there exists a vertex $y \leq x$ in $Q$ such that $X(y) \neq 0$. Therefore in $\bmod _{A}$ we get

$$
P_{y} \leq X \leq R_{i}^{x} \leq P_{x} \leq P_{y} .
$$

Since (2y) is satisfied, then by (1.2) $y$ is not a source in $Q$. Let $z$ be a proper predecessor of $y$ in $Q$. Therefore, $P_{y}$ is a non-directing predecessor of some $R_{j}^{z}$. By $(2 \mathrm{z}), P_{y}$ is not an $A^{z}$-module, contradicting the minimality of $x$.

Then we are in position to repeat the argument given in [2, theorem (2.5)] to prove the existence of a postprojective component. For the sake of completeness we sketch the argument. We construct inductively full subquivers $C_{n}$ of $\Gamma_{A}$ satisfying:

i) $C_{n}$ is finite, connected, contains no oriented cycle and is closed under predecessors.

ii) $\tau_{A}^{-1} C_{n} \cup C_{n} \subset C_{n+1}$.

Then $\cup C_{n}$ forms the wanted postprojective component.

Set $C_{0}=\{S\}$ where $S$ is a simple projective $A$-module. Assume $C_{n}$ to be defined and let $M_{1}, \cdots, M_{t}$ be the modules in $C_{n}$ with $\tau_{A}^{-1} M_{i} \notin C_{n}$. We may assume that $M_{i} \leq M_{j}$ implies $i \leq j$. If $t=0$, set $C_{n+1}=C_{n}$. Otherwise we define full subquivers $D_{i}(0 \leq i \leq t)$ of $\Gamma_{A}$ satisfying $D_{0}=C_{n}, D_{i} \cup\left\{\tau_{A}^{-1} M_{i+1}\right\} \subset D_{i+1}$ and condition (i) imposed on $D_{i}$. Then $D_{n+1}=C_{t}$ will satisfy conditions (i) and (ii). 
Indeed, assume $D_{i}$ is well defined. Take the almost split sequence $0 \rightarrow M_{i+1}$ $\rightarrow X \rightarrow \tau_{A}^{-1} M_{i+1} \rightarrow 0$ and define $D_{i+1}$ as the full subquiver of $\Gamma_{A}$ with vertices $D_{i}$ and all predecessors of $\tau_{A}^{-1} M_{i+1}$. It is enough to show that for each indecomposable direct summand $Y$ of $X$, the set of predecessors $\left\{Z \in \Gamma_{A}: Z \leq Y\right\}$ is finite and formed by directing modules. If $Y$ is not projective, then $\tau_{A} Y \in C_{n}$ whence $Y$ belongs to $D_{i}$ and we are done. If $Y=P_{y}$ is projective, then (3y) is satisfied. By (1.2), we get the result.

1.5. Proof of THE THEOREM. Let $\mathscr{P}$ be a postprojective component of $\Gamma_{A}$. Let $x \in Q_{0}$. If the projective module $P_{x}$ belongs to $\mathscr{P}$, then $(2 \mathrm{x})$ is satisfied. Assume that $P_{x} \notin \mathscr{P}$. We show that $\mathscr{P}$ is formed by $A^{x}$-modules. Let $X \in \mathscr{P}$ and assume $X(y) \neq 0$ for some $y \leq x$ in $Q$. Then $P_{x} \leq P_{y} \leq X$ in $\bmod _{A}$, which implies $P_{x} \in \mathscr{P}$, a contradiction. Hence $\mathscr{P}$ is a postprojective component in $\Gamma_{A^{x}}$ and $R_{i}^{x} \notin \mathscr{P}$ for $1 \leq i \leq n_{x}$, that is $(1 \mathrm{x})$ is satisfied.

Conversely, assume that for each $x \in Q_{0}$, one of the conditions (1x) or $(2 \mathrm{x})$ is satisfied. If for every $x \in Q_{0},(2 \mathrm{x})$ is satisfied then (1.4) implies the result.

Assume that for $x \in Q_{0},(2 \mathrm{x})$ is not satisfied. Choose a minimal such $x$ in the path order in $Q$. By hypothesis $(1 \mathrm{x})$ is satisfied, that is, there is a postprojective component $\mathscr{P}$ of $\Gamma_{A^{x}}$ such that $R_{i}^{x} \notin \mathscr{P}$ for every $1 \leq i \leq n_{x}$. We shall prove that $\mathscr{P}$ is a component of $\Gamma_{A}$. For this purpose it is enough to show that $x$ is a source in $Q$.

Assume $y \leq x$ is a source in $Q$ and $y \neq x$. The minimality of $x$ implies that $(2 y)$ is satisfied. We will show that $(2 x)$ is also satisfied which yields the wanted contradiction. Indeed, let $X$ be a predecessor of $R_{i}^{x}$ in $\bmod _{A}$. Then $X \leq R_{i}^{x} \leq P_{x} \leq P_{y}$, implies that $X$ is a predecessor of $R_{j}^{y}$ for some $1 \leq j \leq n_{y}$. Moreover, since $P_{y}$ is directing in $\bmod _{A}$, then $X$ is an $A^{y}$-module. Thus $\left\{X \in \Gamma_{A}: X \leq R_{i}^{x}\right\}$ is finite and formed by directing modules. Our theorem is proved.

1.6. Corollary. Let $A=k Q / I$ be as above and assume $Q$ is connected. Then all indecomposable projective modules belong to a postprojective component if and only if for every $x \in Q_{0}$ the condition $(2 x)$ is satisfied.

Proof. The "only if" direction is clear. For the converse, assume that for every $x \in Q_{0}$, the condition $(2 \mathrm{x})$ is satisfied. By the theorem there is a postprojective component $\mathscr{P}$ of $\Gamma_{A}$. Clearly we may assume that $Q$ is connected (otherwise we take a postprojective component for each maximal connected full subcategory of $A$ ). Let $x_{0}$ be a sink in $Q$ such that the projective $P_{x_{0}} \in \mathscr{P}$. Let 
$x \in Q_{0}$ and fix a walk $x_{0} \frac{\alpha_{1}}{-} x_{1} \ldots \ldots+\alpha_{s} x_{s}=x$ in $Q$ (that is, each $\alpha_{i}$ is an arrow in $Q$ with some orientation). By induction, we may assume that $P_{x_{s-1}} \in \mathscr{P}$. If $x_{s-1} \stackrel{\alpha_{s}}{\rightarrow} x_{s}$, then $P_{x}$ is a predecessor of $P_{x_{s-1}}$ and $P_{x} \in \mathscr{P}$. Thus, assume that $x_{s} \stackrel{\alpha_{i}}{\rightarrow} x_{s-1}$. Then there is a morphism $f: P_{x_{s-1}} \rightarrow \operatorname{rad} P_{x}$. Since $(2 \mathrm{x})$ is satisfied, then $f$ is a linear combination of compositions of finitely many irreductible maps. Hence $R_{i}^{x} \in \mathscr{P}$ for some $1 \leq i \leq n_{x}$. Thus $P_{x} \in \mathscr{P}$ and we are done.

1.7. EXAMPLES. Consider the algebra $A=k Q / I$ given by the quiver

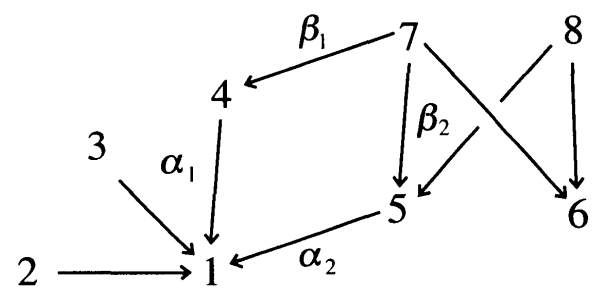

and the ideal $I=\left\langle\alpha_{1} \beta_{1}-\alpha_{2} \beta_{2}\right\rangle$. The quiver $\Gamma_{A}$ has no postprojective component but for every proper full convex subcategory $B$ of $A$, the quiver $\Gamma_{B}$ has a postprojective component. Consider for example $A$ as the one-point extension $A=$ $B[M]$ where $B=A / A e_{7}$ and $M=\operatorname{rad} P_{7}$. Then $B$ is an hereditary algebra and $M=M_{1} \oplus P_{6}$ where $P_{6}$ is a postprojective $B$-module and $M_{1}$ is a regular $B$ module. Therefore $M$ is not directing and both conditions (1x) and (2x) are not satisfied for $x=7$.

It is also interesting to consider $A=C[N]$ where $C=A / A e_{2}$ and $N=\operatorname{rad} P_{2}$. Then $\Gamma_{C}$ has a postprojective component $\mathscr{P}$ and $N=P_{1}$ is an indecomposable module in $\mathscr{P}$. In this case the projective $C$-module $P_{7}$ belongs to $\mathscr{P}$. In section 2 we will consider more carefully this kind of situation.

Finally, we observe that in our example for every convex subcategory $B$ of $A$ (including $B=A$ ), the Auslander-Reiten quiver $\Gamma_{B}$ has a preinjective component.

1.8. Let $A=k Q / I$ be an algebra as above. Let $x \in Q_{0}$ and consider the connected components $Q_{1}^{x}, \cdots, Q_{s_{x}}^{x}$ of the quiver $Q^{x}$ associated with the algebra $A^{x}$. Recall that the vertex $x$ is said to be separating if for each $1 \leq j \leq s_{x}$ the quiver $Q_{j}^{x}$ contains the support of at most one $R_{i}^{x}\left(1 \leq i \leq n_{x}\right)$; thus $s_{x} \geq n_{x}$. The algebra $A$ satisfies the separation condition if all $x \in Q_{0}$ are separating, see $[1,2]$. Observe that with $A$ also $A^{x}$ satisfies the separation condition.

COROLlary [2]. If $A$ satisfies the separation condition, then $\Gamma_{A}$ has a postprojective component. 
Proof. Let $x \in Q_{0}$. Consider $A^{x}=A_{1}^{x} \amalg \cdots \amalg A_{n_{\mathrm{l}}}^{x}$ where $A_{j}^{x}$ is the full convex subalgebra of $A$ with connected quiver $Q_{j}^{x}$. Since also $A_{j}^{x}$ satisfies the separation condition, by induction hypothesis, the Auslander-Reiten quiver of $A_{j}^{x}$ has a postprojective component $\mathscr{P}_{j}$. For each $1 \leq i \leq n_{x}$, we may assume that $R_{i}^{x}$ is an $A_{i}^{x}$-module.

If $R_{i}^{x} \notin \mathscr{P}_{i}$ for some $i$, then $R_{j}^{x} \notin \mathscr{P}_{i}$ for every $1 \leq j \leq n_{x}$. In this case (1x) is satisfied. Otherwise, $R_{i}^{x} \in \mathscr{P}_{i}$ for all $1 \leq i \leq n_{x}$. Then clearly $(2 \mathrm{x})$ is satisfied. Hence (1.3) implies the result.

\section{One-point extensions using postprojective modules.}

2.1. Let $A=k Q / I$ be a finite dimensional $k$-algebra such that $Q$ has no oriented cycles. Let $a$ be a source in $Q$ and consider the quotient $B=A / A e_{a}$. For the $B$-module $M=\operatorname{rad} P_{a}$, we have $A=B[M]$. Let $\mathscr{P}$ be a postprojective component of $\Gamma_{B}$ and assume that all indecomposable direct summands of $M$ belong to $\mathscr{P}$. In this section we consider the problem of when $P_{a}$ belongs to a postprojective component of $\Gamma_{A}$.

We recall that for two $A$-modules $X, Y$ we have $\operatorname{rad}_{A}^{\infty}(X, Y)=\bigcap_{m \geq 0} \operatorname{rad}_{A}^{m}(X, Y)$. We say that an irreducible map $h: X \rightarrow Y$ in $\mathscr{P}$ is $M$-finite if $h \notin \operatorname{rad}_{A}^{\infty}(X, Y)$. An indecomposable $B$-module $X \in \mathscr{P}$ is $M$-finite if there is a walk $M_{i}=$ $X_{0} \stackrel{\alpha_{1}}{\cdots} \ldots \frac{\alpha_{s}}{} X_{s}=X$ in $\mathscr{P}$ (where $M_{i}$ is an indecomposable direct summand of $M$ ) such that each $\alpha_{i}$ is $M$-finite, $1 \leq i \leq s$. Of course, if a map or a module is not $M$-finite we say that it is $M$-infinite.

The following characterization is useful.

LEMMA. Let $h: X \rightarrow Y$ be a map in $\bmod A$ with $X$ and $Y$ indecomposable modules. Then $h \in \operatorname{rad}_{A}^{\infty}(X, Y)$ if and only if there are infinitely many A-modules $L_{n}, n \in N$, without common direct summands and morphisms $f_{n}: X \rightarrow L_{n}, g_{n}$ : $L_{n} \rightarrow Y$ with $g_{n} f_{n}=h$.

Proof. Assume that $h \in \operatorname{rad}^{\infty}(X, Y)$. We construct the modules $L_{n}$ inductively. For $n=1$, we set $L_{1}=X$. Assume we have already constructed $L_{1}, \cdots, L_{n}$ as in the statement. Let $m$ be the maximal of $\operatorname{dim}_{k} C$ for $C$ an indecomposable direct summand of some $L_{i}, 1 \leq i \leq n$. By the Harada-Sai Lemma, there is a number $N(m)$ such that for every chain $C_{1} \rightarrow C_{2} \rightarrow \cdots \rightarrow C_{s}$ of non isomorphisms between indecomposable modules with $\operatorname{dim}_{k} C_{i} \leq m+1$, if $s \geq N(m)$, then the composition of the chain is zero. Since $h \in \operatorname{rad}_{A}^{N(m)}(X, Y)$, then $h$ may be 
written as a linear combination $h=\sum_{i=1}^{r} h_{i}$, where $h_{i}$ is composition of $N(m)$ nonisomorphisms between indecomposable modules. Therefore each $h_{i}$ factorizes through some indecomposable module $Z_{i}$ with $\operatorname{dim}_{k} Z_{i} \geq m+1,1 \leq i \leq r$. We can define $L_{n+1}=\oplus_{i=1}^{r} Z_{i}$.

For the converse, define inductively the finite set of indecomposable modules $X^{(n)}$ in the following way. The set $X^{(1)}$ is formed by those indecomposable modules which are direct summands of the module $Z$, where $X \rightarrow Z$ is a source map in the category $\bmod A$. If $X^{(n)}$ is defined, then $X^{(n+1)}$ is formed by those modules in $Z^{(1)}$ for $Z$ in $X^{(n)}$. For any $n$, choose an $m$ such that the module $L_{m}$ has no direct summands in $X^{(n)}$. Consider the factorization $h=g_{m} f_{m}$ with $f_{m}: X \rightarrow L_{m}, g_{m}: L_{m} \rightarrow Z$. Using the properties of source maps, we get that $f_{m}$ lies in $\operatorname{rad}_{A}^{n}\left(X, L_{m}\right)$. Hence $h \in \operatorname{rad}_{A}^{\infty}(X, Z)$.

2.2. Consider the directed vector space category $\operatorname{Hom}_{A}(M, \mathscr{P})$, see $[3,6]$. Denote by $|X|=\operatorname{Hom}_{A}(M, X), X \in \mathscr{P}$. Then the full subcategory of $\operatorname{Hom}_{A}(M, \mathscr{P})$ whose objects are those $|X| \neq 0$ with $X \in \mathscr{P}$, form a poset $\mathscr{P}_{M}$. Indeed, $|X| \leq|Y|$ in $\mathscr{P}_{M}$ implies that $X \leq Y$ in $\mathscr{P}$.

A subposet $\mathscr{V}$ of $\mathscr{P}_{M}$ is said to be of finite type if for each $|X| \in \mathscr{V}$, $\operatorname{dim}_{k}|X| \leq 1$ and $\mathscr{V}$ does not contain as a full subposet one of the posets $(1,1,1,1)$, $(2,2,2),(1,3,3),(1,2,5)$ or $(N, 4)$ of Kleiner's list.

If $\mathscr{P}_{M}$ is representation-infinite there is a infinite family of triples $Y_{\lambda}=\left(V, Y, \gamma_{\lambda}: V \rightarrow \operatorname{Hom}_{B}(M, Y)\right)$ where $V \in \bmod _{k}, Y$ is a $B$-module whose indecomposable direct summands $X$ have $|X| \in \mathscr{P}_{M}$ and $\gamma_{\lambda}$ is linear, corresponding to indecomposable pairwise non-isomorphic $A$-modules. A module $X \in \mathscr{P}$ is said to be $M$-representation-infinite if there are infinitely many pairwise nonisomorphic indecomposable $A$-modules of the form $\left(V, Y, \gamma: V \rightarrow \operatorname{Hom}_{B}(M, Y)\right)$ where $V \in \bmod _{k}, Y$ is a $B$-module with $X$ as a direct summand and $\gamma$ is linear.

LEMMA. Let $h: X \rightarrow Y$ be an irreducible map in $\mathscr{P}$. Then $h$ is $M$-infinite if and only if the following two conditions hold

i) $X$ is $M$-representation-infinite;

ii) there is a morphism $0 \neq g \in \operatorname{Hom}_{B}(M, X)$ with $h g=0$.

Proof. First assume that $h \in \operatorname{rad}_{A}^{\infty}(X, Y)$. Then there are infinitely many $A$ modules $L_{n}=\left(V_{n}, Z_{n}, \gamma_{n}: V_{n} \rightarrow \operatorname{Hom}_{B}\left(M, Z_{n}\right)\right), \quad n \in N$ without common direct summands and morphisms $f_{n}: X \rightarrow L_{n}, g_{n}: L_{n} \rightarrow Y$ with $g_{n} f_{n}=h$. Fix $n \in N$ and let $Z_{n}=X^{a} \oplus Y^{b} \oplus Z_{n}^{\prime}$ be such that $X$ and $Y$ are not summands of $Z_{n}^{\prime}$. The following diagrams commute: 


$$
\left(\begin{array}{c}
\lambda_{i} \\
h_{j}^{\prime} \\
*
\end{array}\right)=\left.f_{n}\right|_{Z_{n}=X^{a} \oplus Y^{b} \oplus Z_{n}^{\prime}} ^{\stackrel{h}{\longrightarrow}} \overbrace{}^{Y} g_{n}=g\left(h_{i}^{\prime \prime}, \mu_{j}, *\right)
$$

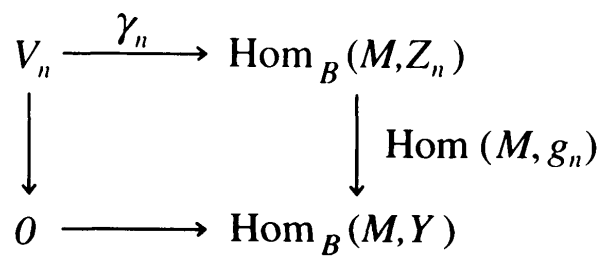

with $\lambda_{i} \in k, h_{i}^{\prime \prime} \in \operatorname{Hom}_{B}(X, Y)(1 \leq i \leq a), \mu_{j} \in k, h_{j}^{\prime} \in \operatorname{Hom}_{B}(X, Y)(1 \leq j \leq b)$. Without loss of generality we may assume that $V_{n} \neq 0$ and $(0, X, 0),(0, Y, 0)$ are not direct summands of $L_{n}$. First we show that $\mu_{j}=0(1 \leq j \leq b)$. Otherwise there is some $0 \neq v \in V_{n}$ and $\gamma_{n}(v)=\left(v_{i}^{\prime}, v_{j}^{\prime \prime}, *\right)$ with $v_{j_{0}}^{\prime \prime} \neq 0$ and Hom $\left(M, \mu_{j_{0}}\right)\left(v_{j_{0}}^{\prime \prime}\right) \neq 0$ for some $j_{0}$, a contradiction. Since $h$ is irreducible as a $B$-morphism, then $a>0, \lambda_{i_{0}} \neq 0$ and $h_{i_{0}}^{\prime \prime}$ is a non-zero multiple of $h$ for some $1 \leq i_{0} \leq a$. This shows (i). Moreover, there is some $0 \neq \omega \in V_{n}$ with $\gamma_{n}(\omega)=\left(\omega_{i}^{\prime}, \omega_{j}^{\prime \prime}, *\right)$ and $0 \neq \omega_{i_{0}}^{\prime} \in \operatorname{Hom}_{B}(M, X)$. Therefore $\omega_{i_{0}}^{\prime} h_{i_{0}}^{\prime \prime}=\operatorname{Hom}\left(M, h_{i_{0}}^{\prime \prime}\left(\omega_{i_{0}}^{\prime}\right)\right)=0$ and condition (ii) holds.

For the converse, consider an infinite family $L_{n}=\left(V_{n}, Z_{n}, \gamma_{n}\right)$ of pairwise nonisomorphic indecomposable $A$-modules $(n \in N)$ such that $X$ is a direct summand of $Z_{n}$. Let $Z_{n}=X \oplus Z_{n}^{\prime}$ and $\sigma_{n}: X \rightarrow Z_{n}$ be the canonical inclusion. Assume first that $\operatorname{dim}_{k}|X|=1$. Then for the $A$-morphism $g_{n}=\left(0, h \pi_{n}\right): L_{n} \rightarrow Y$ where $\pi_{n}: Z_{n} \rightarrow X$ is the canonical projection, we get $g_{n} \sigma_{n}=h$. This may only happen if $h \in \operatorname{rad}_{A}^{\infty}(X, Y)$. Now, assume that $\operatorname{dim}_{k}|X| \geq 2$ and take $b \in \operatorname{Hom}_{B}(M, X)$ such that $g, b$ are linearly independent. Then we may choose $Z_{n}=X \oplus X, V=k$ and $\gamma_{n}: k \rightarrow \operatorname{Hom}_{B}(M, X)^{2}, 1 \mapsto\left(\lambda_{n} g, b\right)$ for some $\lambda_{n} \neq 0$. Again, if $g_{n}=\left(0, h \pi_{n}\right)$ : $L_{n} \rightarrow Y$ where $\pi_{n}: X \oplus X \rightarrow X$ is the first canonical projection, we get $g_{n} \sigma_{n}=h$. We are done.

2.3. The main result in this section is the following:

THEOREM. Let $A=B[M]$ be a one-point extension algebra with $M=\operatorname{rad} P_{a}$ for a source a of $Q$. Assume that all indecomposable direct summands of $M$ belong to a postprojective component $\mathscr{P}$ of $\Gamma_{B}$.

If $P_{a}$ belongs to a postprojective component of $\Gamma_{A}$ then the following conditions hold:

a) $M$ is directing;

b) for every irreducible map $h: X \rightarrow Y$ in $\mathscr{P}$ such that $Y$ is $M$-finite, then $h$ is $M$-finite;

c) for every indecomposable projective B-module $P_{y} \in \mathscr{P}$ which is M-finite, the set of predecessors of $P_{y}$ in $\Gamma_{A}$ is finite and formed by directing modules.

Conversely, if conditions $(a)$ and $(c)$ hold, then $P_{a}$ belongs to a postprojective component of $\Gamma_{A}$. 
Proof. Assume first that $\mathscr{P}^{\prime}$ is a postprojective component of $\Gamma_{A}$ containing $P_{a}$. Therefore $M$ is directing.

Let $Y \in \mathscr{P}$ be $M$-finite, we show that $Y \in \mathscr{P}^{\prime}$. Indeed, consider a chain of irreducible maps $M \stackrel{\alpha_{1}}{\longrightarrow} X_{1} \frac{\alpha_{2}}{-} X_{2}-\cdots \frac{\alpha_{s}}{-} X_{s}=Y$ with $\alpha_{i}$ being $M$-finite. By induction we may assume that $X_{s-1} \in \mathscr{P}^{\prime}$. If $X_{s} \stackrel{\alpha_{s}}{\rightarrow} X_{s-1}$, then clearly $X_{s} \in \mathscr{P}^{\prime}$. If $X_{s-1} \stackrel{\alpha_{s}}{\rightarrow} X_{s}$ and $X_{s} \notin \mathscr{P}^{\prime}$, then $\alpha_{s} \in \operatorname{rad}_{A}^{\infty}\left(X_{s-1}, X_{s}\right)$, which is a contradiction. Therefore $Y \in \mathscr{P}^{\prime}$.

We show (b): let $h: X \rightarrow Y$ be an irreducible map in $\mathscr{P}$ and assume $Y$ to be $M$-finite. Then $Y \in \mathscr{P}^{\prime}$ and also $X \in \mathscr{P}^{\prime}$. Since $\mathscr{P}^{\prime}$ is postprojective, $h \notin \operatorname{rad}_{A}^{\infty}(X, Y)$. And (c): let $P_{y} \in \mathscr{P}$ be $M$-finite. Then $P_{y} \in \mathscr{P}^{\prime}$ and therefore $P_{y}$ has only finitely many predecessors in $\Gamma_{A}$, all of them directing.

For the converse we proceed as in (1.4) to construct a postprojective component $\mathscr{P}^{\prime}$ of $\Gamma_{A}$. Indeed, we define inductively full subquivers $C_{n}$ of $\Gamma_{A}$ satisfying: (i) $C_{n}$ is finite, connected, contains no oriented cycle and is closed under predecessors and (ii) $\tau_{A}^{-1} C_{n} \cup C_{n} \subset C_{n+1}$.

Let $S$ be a simple projective in $\mathscr{P}$, then set $C_{0}=\{S\}$. Assume $C_{n}$ is well defined and let $X_{1}, \cdots, X_{t}$ be those modules in $C_{n}$ with $\tau_{A}^{-1} X_{i} \notin C_{n}$, numbered in such a way that $i<j$ whenever $X_{i} \leq X_{j}$. Define $D_{0}=C_{n}, D_{i+1}$ as the full subquiver of $\Gamma_{A}$ consisting of $D_{i}$ and the predecessors of $\tau_{A}^{-1} X_{i+1}$ and $C_{n+1}=D_{t}$. It is enough to show inductively that $D_{i}$ satisfies condition (i) above. Consider the AuslanderReiten sequence $0 \rightarrow X_{i+1} \rightarrow X \rightarrow \tau_{A}^{-1} X_{i+1} \rightarrow 0$ and assume that $D_{i}$ satisfies (i). We shall prove that each indecomposable direct summand $Y$ of $X$ has only finitely many predecessors, all of them directing.

We first show the following: let $\left(V, N, \gamma: V \rightarrow \operatorname{Hom}_{B}(M, N)\right)$ be an indecomposable module in $D_{i}$, then every indecomposable direct summand $N^{\prime}$ of $N$ belongs to $\mathscr{P}$ and is $M$-finite. We proceed by induction on the path order in $D_{i}$ (which satisfies (i)). As a first case, assume that $V=0$. If $N=P_{y}$ is projective, then every direct summand $R_{i}^{y}$ of $\operatorname{rad} P_{y}$ belongs to $\mathscr{P}$ and is $M$-finite. Therefore $N \in \mathscr{P}$. Moreover, since the canonical inclusion $R_{i}^{y} \rightarrow N$ is not in $\operatorname{rad}_{A}^{\infty}\left(R_{i}^{y}, N\right)$, then $N$ is $M$-finite. If $N$ is not projective, consider the Auslander-Reiten sequence $0 \rightarrow \tau_{B} N \stackrel{\sigma}{\rightarrow} E \rightarrow N \rightarrow 0$ in $\bmod _{B}$ and the corresponding sequence $0 \rightarrow \overline{\tau_{B} N} \rightarrow \bar{E} \rightarrow N \rightarrow 0$ in $\bmod _{A}$, where $\bar{E}=\left(\operatorname{Hom}_{B}\left(M, \tau_{B} N\right), E, \operatorname{Hom}_{B}(M, \sigma)\right)$. Since the indecomposable direct summands of $\bar{E}$ belong to $D_{i}$ by induction hypothesis we get that the indecomposable direct summands of $E$ belong to $\mathscr{P}$ and are $M$-finite. Hence $N \in \mathscr{P}$. Moreover, since $N$ is in $D_{i}$, it has only finitely many predecessors and therefore any irreducible map $E_{i} \rightarrow N$ in $\mathscr{P}$ is $M$-finite. For the second case, assume that $V \neq 0$ and take an indecomposable direct summand $N^{\prime}$ of $N$. Hence $\operatorname{Hom}_{B}\left(M, N^{\prime}\right) \neq 0$. Suppose that $N^{\prime}$ is not in $\mathscr{P}$, then $\operatorname{rad}_{B}^{\infty}\left(M, N^{\prime}\right) \neq 0$ 
and $N^{\prime}$ has infinitely many predecessors. The same happens to $(V, N, \gamma)$ which contains $\left(0, N^{\prime}, 0\right)$. A contradiction showing that $N^{\prime} \in \mathscr{P}$. In the same way $N^{\prime}$ is $M$-finite.

Now we continue the main line of the proof. Let $Y$ be an indecomposable direct summand of $X$. If $Y$ is not projective, then $Y$ belongs to $D_{i}$ and we are done. Assume that $Y$ is projective. Consider first the case $Y=P_{a}$. By (a), $P_{a}$ is directing and therefore the predecessors of $P_{a}$ in $\bmod _{A}$ are $B$-modules and are predecessors of some direct summand $M_{i}$ of $M=\operatorname{rad} P_{a}$ in $\bmod _{B}$. Since every $M_{i}$ belongs to $\mathscr{P}$, then $Y=P_{a}$ has only finitely many (all directing) predecessors. Finally assume that $Y=P_{y}$ for some $y \neq a$. Let $R_{\mathrm{i}}^{y}$ be a direct summand of $\operatorname{rad} P_{y}$ belonging to $D_{i}$. By the claim shown above, $R_{i}^{y} \in \mathscr{P}$ and $R_{i}^{y}$ is $M$-finite. Therefore $P_{y} \in \mathscr{P}$ and it is also $M$-finite. By hypothesis (c), $Y=P_{y}$ has only finitely many (all directing) predecessors in $\Gamma_{A}$. This finishes our proof.

2.4. We consider again the example (1.7). With the notation introduced there $A=\mathrm{C}[N]$ where $N=P_{1}$ is simple projective. We sketch part of the postprojective component $\mathscr{P}$ of $\Gamma_{C}$ where $N$ lies.

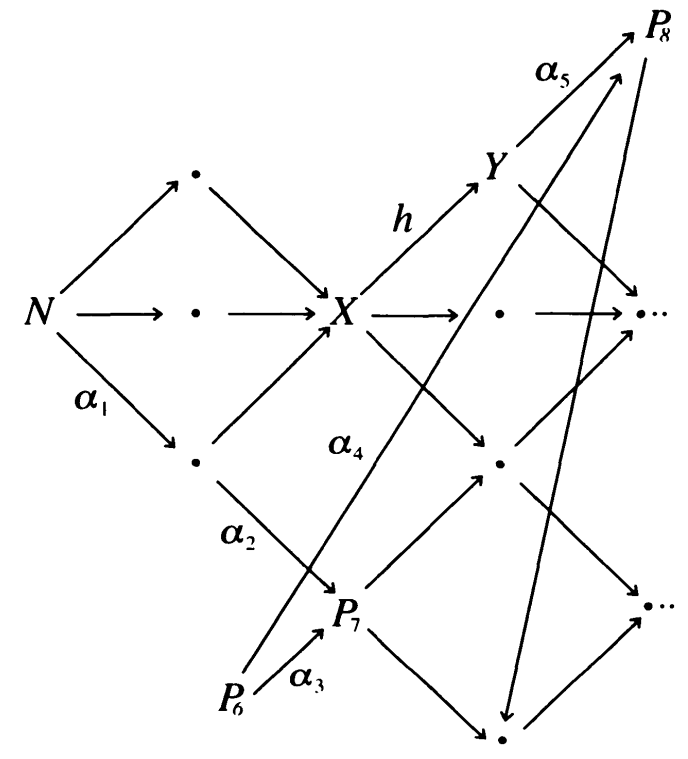

The walk $\alpha_{5}^{-1} \alpha_{4} \alpha_{3}^{-1} \alpha_{2} \alpha_{1}$ from $N$ to $Y$ is formed by $N$-finite irreducible maps, therefore $Y$ is $N$-finite. On the other hand, $\operatorname{dim}_{k} \operatorname{Hom}_{C}(N, X)=2$ and $\operatorname{dim}_{k} \operatorname{Hom}_{C}(N, Y)=1$, therefore by (2.2), $h$ is not $N$-finite. By (2.3), $P_{2}$ does not belong to a postprojective component in $\Gamma_{A}$.

\section{Some quadratic conditions.}

3.1. In this section we consider again the situation of section 2 and we find 
some necessary conditions for the existence of a postprojective component in $\Gamma_{A}$ containing the projective module corresponding to the extension vertex. These conditions are expressed by the values of certain quadratic forms.

Let $A=B[M]$ be a one-point extension of the algebra $B$ by the module $M=\operatorname{rad} P_{a}$. Let $M=\oplus_{i=1}^{s} M_{i}$ be the indecomposable decomposition of $M$. Consider the Euler form associated with $B$ :

$$
\langle\underline{\operatorname{dim}} X, \underline{\operatorname{dim}} Y\rangle_{B}=\sum_{j=0}^{\infty}(-1)^{j} \operatorname{dim}_{k} \operatorname{Ext}_{B}^{j}(X, Y),
$$

where $\underline{\operatorname{dim} X}$ is the element of the Grothendieck group $K_{0}(B)$ corresponding to $X$. See [3].

For different $i, j \in\{1, \cdots, s\}$, we define the quadratic form

$$
q_{i j}(\omega)=\left\langle\omega, \underline{\operatorname{dim}} M_{i}\right\rangle_{B}\left\langle\omega, \underline{\operatorname{dim}} M_{j}\right\rangle_{B} .
$$

3.2. Proposition. Let $\mathscr{P}_{1}, \cdots, \mathscr{P}_{m}$ be the postprojective components of $\Gamma_{B}$ and assume that $m \geq 1$. Suppose that $\Gamma_{A}$ has a postprojective component, then there exists a component $\mathscr{P}_{t}$ such that for every two different $i, j, \in\{1, \cdots, s\}$ and every $X \in \mathscr{P}_{t}$ with $\operatorname{projdim}_{B} X \leq 1$, we have

$$
q_{i j}(\underline{\operatorname{dim} X}) \geq 0
$$

ProOF. First assume that for some $t \in\{1, \cdots, m\}$, there is no $M_{i}$ belonging to $\mathscr{P}_{t}$. Take $X \in \mathscr{P}_{t}$ with proj $\operatorname{dim}_{B} X \leq 1$, then

$$
\left\langle\underline{\operatorname{dim}} X, \underline{\operatorname{dim}} M_{i}\right\rangle_{B}=\operatorname{dim}_{k} \operatorname{Hom}_{B}\left(X, M_{i}\right)-\operatorname{dim}_{k} \operatorname{Ext}_{B}^{1}\left(X, M_{i}\right) .
$$

Since $M_{i} \notin \mathscr{P}_{t}$, then $\operatorname{Ext}_{B}^{1}\left(X, M_{i}\right)=0$ and $\left\langle\underline{\operatorname{dim}} X, \underline{\operatorname{dim}} M_{i}\right\rangle_{B} \geq 0$. This shows that $q_{i j}(\underline{\operatorname{dim} X}) \geq 0$ for any two $i, j \in\{1, \cdots, s\}$.

In the other case, choose $t=1$. Take $i, j \in\{1, \cdots, s\}$ different and $X \in \mathscr{P}_{1}$ with proj $\operatorname{dim}_{B} X \leq 1$. Assume that

$$
\left\langle\underline{\operatorname{dim}} X, \underline{\operatorname{dim}} M_{i}\right\rangle_{B}<0<\left\langle\underline{\operatorname{dim}} X, \underline{\operatorname{dim}} M_{j}\right\rangle_{B} .
$$

Since proj $\operatorname{dim}_{B} X \leq 1$, this implies that $\operatorname{Ext}_{B}^{1}\left(X, M_{i}\right) \neq 0 \neq \operatorname{Hom}_{B}\left(X, M_{j}\right)$. The Auslander-Reitern formula gives $0 \neq D \operatorname{Ext}_{B}^{1}\left(X, M_{i}\right) \cong \overline{\operatorname{Hom}}_{B}\left(M_{i}, \tau_{B} X\right)$ (see [3]). Therefore there is a path in $\Gamma_{B}, M_{i} \leq \tau_{B} X \leq X \leq M_{j}$. By (1.2), $P_{a}$ is not directing. Let $\mathscr{P}$ be a postprojective component of $\Gamma_{A}$. Since each $\mathscr{P}_{\ell}$ for $1 \leq \ell \leq m$, contains a summand of $M$, then $\mathscr{P} \neq \mathscr{P}_{\ell}$. Therefore $\mathscr{P}$ is not a component of $\Gamma_{B}$. Hence it contains a module $Y \in \mathscr{P}$ with $0 \neq Y(a)=\operatorname{Hom}_{A}\left(P_{a}, Y\right)$. This implies that $P_{a} \in \mathscr{P}$. But then $P_{a}$ should be directing, a contradiction. We are done.

3.3. We come back to our example (1.7) now considering $A=B[M]$ where $M$ 
$=\operatorname{rad} P_{7}$. Thus $M=M_{1} \oplus M_{2}$, where

$$
\underline{\operatorname{dim}} M_{1}=(1,0,0,1,1,0,0) \text { and } \underline{\operatorname{dim}} M_{2}=(0,0,0,0,0,1,0)
$$

in $K_{0}(B)$. There is a unique postprojective component $\mathscr{P}_{1}$ of $\Gamma_{B}$ which has the shape

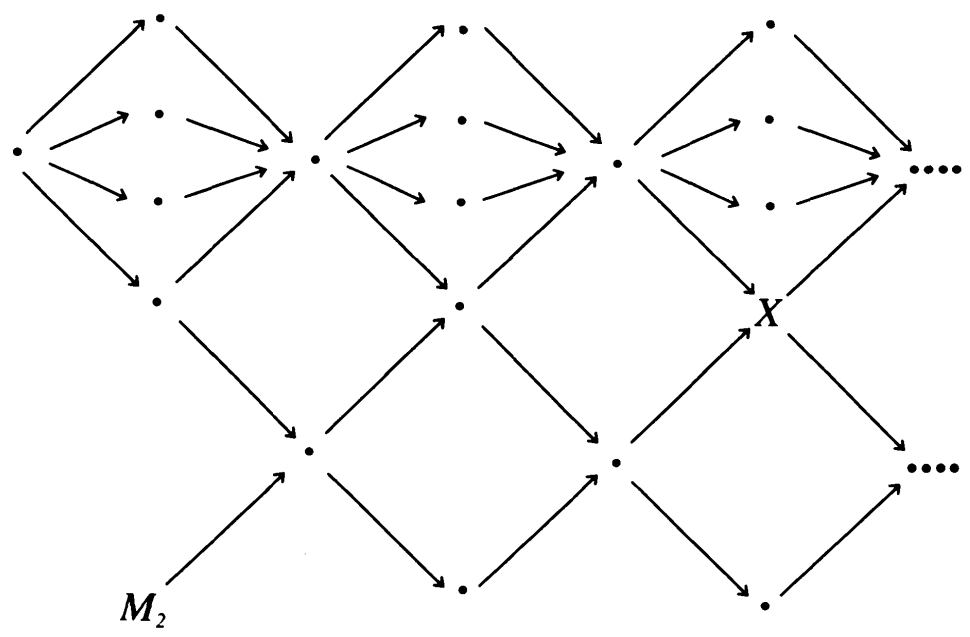

where $\underline{\operatorname{dim}} X=(6,2,2,2,3,0,1) \in K_{0}(B)$ and clearly proj $\operatorname{dim}_{B} X \leq 1$.

We have

$$
\left\langle x, \underline{\operatorname{dim}} M_{1}\right\rangle_{B}=x_{1}-x_{2}-x_{3}-x_{7} \text { and }\left\langle x, \underline{\operatorname{dim}} M_{2}\right\rangle_{B}=x_{6}-x_{7} .
$$

Hence $q_{12}(\underline{\operatorname{dim} X})=-1$. The quiver $\Gamma_{A}$ has no postprojective component (as we already knew).

\section{References.}

[ 1 ] Bautista, R., Larrión, F. and Salmerón, L.: On simply connected algebras. J. London Math. Soc. (2) 27 (1983), 212-220.

[ 2 ] Bongartz, K.: A criterion for finite representation type. Math. Ann. 269 (1984), 1-12.

[ 3 ] Gabriel, P. and Roiter, A. V.: Representation of finite-dimensional algebras. Algebra VIII Encyclopaedia of Math. Sc. Vol. 73 (1992).

[ 4 ] Happel, D. and Ringel, C. M.: Directing projective modules. Archiv. Math. 60 (1993) 237-243.

[ 5 ] Kasjan, S. and de la Peña, J. A.: Constructing the postprojective components of an algebra. J. Algebra. 179 (1996), 793-807.

[6] Ringel, C. M.: Tame algebras and integral quadratic forms. Lecture Notes in Mathematics 1099, Springer, Berlin (1984).

[ 7 ] Skowronski, A. and Wenderlich, M.: Artin algebras with directing indecomposable projectives. J. Algebra. 165 (1994), 507-530.

[8] Strauss, H.: The perpendicular category of a partial tilting module. J. Algebra 144 (1991) 4366. 
P. Dräxler

Fakultät Füf Mathematik

Universität Bielefeld

D-4800 Bielefeld

Germany

José Antonio de la Peña

Instituto de Matemáticas, UNAM

México 04510, D.F.

México 